\title{
Defining Formal Letter Similarity for Purposes of Accessing Web Information by Users with Dyslexia and Other Reading Disorders
}

\author{
Tereza Pařilová, Eva Hladká, and Jaroslav Bayer
}

\begin{abstract}
In many papers, dyslexia is identified as a specific learning difficulty (SpLD). However, reading disorders do not have to be only due to dyslexia. Development of technologies for users with reading or symbol disorder is neglected due to the statistically inconsistent number of users diagnosed across languages, or because of the dependency of the technology on specific language principles. We are developing an application that will allow Czech speaking users with reading disorders to access web information better, which also means easier socialization and access to education. For such an application, we need to determine what the problem in text is for (not only diagnosed) dyslexic users and how we can find it. Therefore, a simple formal methodology for identifying visual similarity of letters is needed. The LAP algorithm introduced in this paper will help to find possible similarity view as users with reading disorders have. The application built upon this will be used for allowing dyslexic users to better access text information from web and also for users with temporary neurological disorders as a result of a disease, trauma or operation.
\end{abstract}

Index Terms - Web accessibility, dyslexia, design, language accessibility, dyslexia, similarity search.

\section{INTRODUCTION}

Dyslexia is a cognitive disorder that affects people across the world. There are different opinions about its origin [1], [2]. It is probably related with coordination between left and right hemisphere, the corpus callosum, where visual information between hemispheres are exchanged [3]. A general problem of users with dyslexia is that they cannot correctly or in usual time ,decrypt“" letters in text, and thus decrypting whole words, potentially sentences and paragraphs, is hard or impossible. Dealing with alphabet, dyslexia is language depended.

Different language systems (families) may need its own technology for users with dyslexia, which can solve the problems stated above. Besides English, which is one of the most spoken languages in the world, Athanaselis et al., Engel-Eldar and Rosenhouse, Kenvinde et al. or Rello and Baeza-Yates show that attempts to develop technology accommodating dyslexics' needs come from Greece, Spain and Israel [4]-[7]. However, designs of such applications hardly make text easier to read. For example Spanish applications are almost solely in the form of games in which

Manuscript received July 16, 2015; revised November 15, 2015.

All authors are with the Masaryk University/Faculty of Informatics, Brno, Czech Republic (e-mail: \{parilova, eva, 72873\}@mail.muni.cz). children are asked to fill out missing letters [8]. It must be stressed that this practicing is more close to proving dysgraphia, not dyslexia, although these disorders are very often diagnosed together (not necessarily). Dyslexia does not belong to major diseases that would be comparable, for example, to blindness; users with dyslexia, however, deal with their own discomfort and they should not be omitted by designers and developers, nor researchers and society.

\section{THE NEEDS OF THE TEXT}

As was stated before, the main problem that makes reading hard for users with dyslexia is decrypting letters due to visual disorder based in the brain. Therefore, to dyslexic users, some letters look very similar and in some cases they are not even able to distinguish between them (they are indecipherable, "IDP"). The letters may look like reversed in any way, have very similarly positioned edges, curves, etc. [9]. If more of such letters are next to or closed to each other in a word, they might be easily confused and the word does not make sense in the context of the text, especially when more words contain these IDP letters. As a result, text can be confusing, misunderstood, and, in the worst case, not understandable.

The text needs graphical accommodation that in a correct way visually fragments the word, but the word also needs to stay consistent with no white spaces between the letters [10], [11]. First, the similarity of letters needs to be defined formally to build a dyslexic model of similarity. There are two ways of selecting letters that together or close to each other can be seen as similar by dyslexic users; (a) by testing users with dyslexia, (b) by calculating the similarity formally.

\section{RELATED WORK}

Graphical similarity is generally used in many areas of research, such as authorship identification, large database searching, etc. [12], [13]. Up to date, more than 70 studies using different methodologies have been carried out, most of them are, however, years old and applied habitually to English.

Object similarity is generally determined by different possible methods. To move away specifically from the use in dyslexia research, the comparisons of graphical objects are done using e. g. Scalable Invariant Feature Transform (SIFT) or Speeded-Up Robust Features (SURF) algorithms which are based on vector calculation [14], [15].

Another common approach to similarity detection is 
through machine learning algorithm, for example Support vector Machine (SVM) or k-Nearest Neighbour (k-NN). Artificial neural nets (ANN) or Edit Distance Approach (EDA) are other solutions [16], [17].

To our knowledge there are no detections of letter similarity for the purpose of dyslexia. There have been several papers on text accessibility for users with dyslexia [18]-[20], nevertheless none of the approaches solve the original problem of dyslexia, therefore are not considered to be auxiliary materials on which a web accessibility approach could be built.

\section{DESIGN OF LETTER Similarity TESTING}

\section{A. Testing Dyslexic Users}

Ten users were tested in pilot study. Users were asked to mark letters that seem them too similar that they confuse them within a word (sentence). The results are shown in Fig. 1. Demographic data of the volunteers is in Table I.

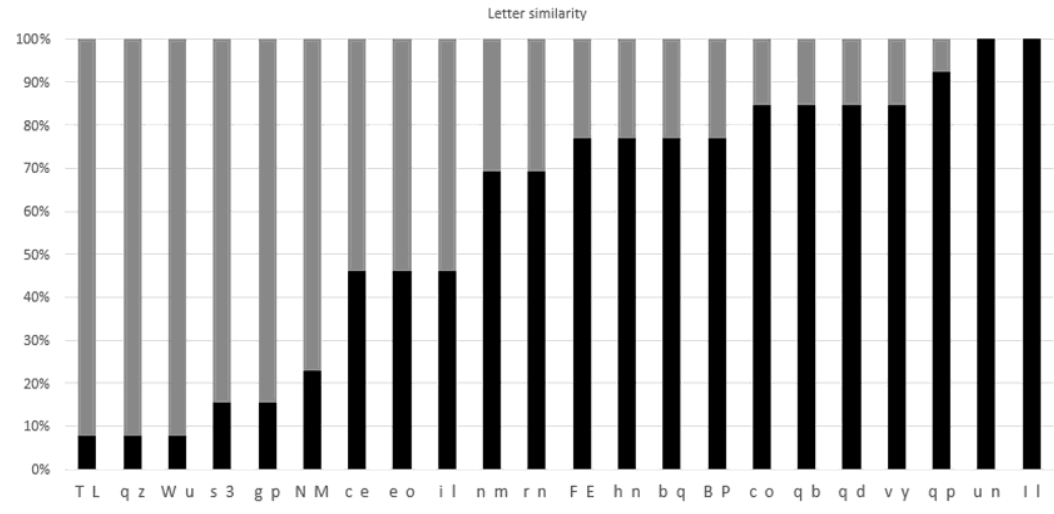

Fig. 1. The ratio of similarly looking letters to users with dyslexia.

TABLE I: DEMOGRAPHIC INFORMATION OF USERS UNDERTAKING THE

\begin{tabular}{|c|c|c|} 
LETTER SIMILARITY TEST \\
\hline Volunteer & Sex & Age \\
\hline 1 & F & 37 \\
\hline 2 & M & 26 \\
\hline 3 & F & 18 \\
\hline 4 & F & 18 \\
\hline 5 & F & 46 \\
\hline 6 & M & 33 \\
\hline 7 & M & 54 \\
\hline 8 & F & 56 \\
\hline 9 & F & 38 \\
\hline 10 & F & 20 \\
\hline
\end{tabular}

\section{B. Formalizing Similarity}

Besides ways of observing critical points in text through eye tracking [21], [22] or simply asking users with dyslexia to label letters in text that make problems while reading, there should be also formal proof of similarity and comparison with empirical data. Dyslexia is found to be very individual in some cases and there need to be a way of establishing which letters (letter patterns) and why causes typical (general) problems. There are several ways we can formulate similarity between letters.

Let's assume the letter is a graphical object that has its specific attributes, so each different letter has its own identification criteria enabling us to determine whether the object is a letter, and if so, then which one [23]. For such determination, we can use attributes that computer vision algorithms work with, such as blobs, corners or edges, and combinations of these. In our research, we chose two methods for formalising and confirming similarities that were identified by our volunteering users. The first one is based on pixels and matrices; we designed it for our purposes and call it LAP (Overlapping algorithm). The second one is SIFT algorithm that uses feature descriptions to compare two pictures [24], [25]. Other control algorithms could be used as well.

1) LAP algorithm

For pilot testing, we introduce an algorithm that works with pixels on matrix. We take combinations of Latin alphabet letters, monospaced sans-serif Courier font with size set as 30x50 points, and rasterized them. The raster was considered black-and-white with two states of pixels - state 1 for 'switched on' (existing pixel), state 0 for 'switched off' (non-existing pixel).

We match them on matrices in different shapes, which makes 3250 possible combinations: let's have a set of Latin alphabet symbols excluded special characters used in Czech language (these characters comprise of hooks above regular letter $\check{c}, \check{z}, \check{e}, \check{s}$ etc. and a kind of dash above the letter $\grave{i}, e ́, a ́$, $\dot{y}$ etc. A specific letter $c h$ was also excluded because principally it consists of two letters, Czech alphabet however have $c h$ as one letter).

Then, $\{a, b, c, d \ldots \ldots z\}=S ;|\mathrm{S}|=26$, i.e. the set of the alphabet has 26 letters. There are five rotations used for every letter. Total number of letter combination for comparison is then equal to formula (1).

$$
\{|S| *[(|S|-1) * 5]\}
$$

In spatial rotations it comprises (a) $180^{\circ}$ on axis $x$, (b) $180^{\circ}$ on axis $y$ and (c) $90^{\circ}, 180^{\circ}$ and $270^{\circ}$ on axis $z$. Rotations of letters $p$ and $q$ for comparison can be seen on Fig. 2.

\begin{tabular}{|c|c|c|c|c|c|}
\hline Letters $/$ Botations & $\mathbf{x}=\mathbf{1 8 0 ^ { \circ }}$ & $\mathbf{y}=\mathbf{1 8 0 ^ { \circ }}$ & $\mathbf{z}=\mathbf{9 0 ^ { \circ }}$ & $\mathbf{z}=\mathbf{1 8 0 ^ { \circ }}$ & $\mathbf{z}=\mathbf{2 7 0 ^ { \circ }}$ \\
\hline $\mathrm{q}$ & $\mathrm{p}$ & $\mathrm{d}$ & $\sigma$ & $\mathrm{b}$ & $\varrho$ \\
\hline $\mathrm{p}$ & $\mathrm{q}$ & $\mathrm{b}$ & $\varrho$ & $\mathrm{d}$ & ○ \\
\hline
\end{tabular}

Fig. 2. Rotations of lower case letters $p$ and $q$. 
Rotations of letter $p$ are in black, rotations of letter $q$ are in red; different rotations of these different letters however make similar-looking letters; this represents the problem of dyslexic users; as a matter of fact, the letter looking like $b$ is not $b$ but $p$ rotated $180^{\circ}$ on axis $y$ while $q$ rotated $180^{\circ}$ on axis $z$.

After then, we calculate distance from on/off pixels in these variants: (a) number of pixels with value 1 on the same coordinates; (b) number of pixels with equal value; (c) number of pixels with value 1 of compared letter minus number of pixels with value 1 on the same coordinates (here we break the axiom of metric symmetry). We get results telling us how many pixels are not overlapped and therefore what percentage of the letters in the pair is similar. Compared to soft data from dyslexic users and the questionnaire, we determine what should be the threshold for deciding similarity in percentage.

As a programming language we used Perl, the rasterization was obtained through data serialization from Canvas HTML5. We consider these steps as the fastest and easiest solution for our problem.

After the counting, we got result that is shown in Table II. Letters $p, q$ and $z$ are again shown for better comparison with results from SIFT. Results in graphic are on Fig. 3.

TABLE II: THE MOST SIMILAR LETTERS FROM LEFT TO RIGHT

\begin{tabular}{|l|l|}
\hline Letter & Similarity calculated \\
\hline $\mathrm{p}$ & pbogqdenhasucmwykzrxtvifjl \\
\hline $\mathrm{q}$ & qgdoaepbcuhnsmwzkyxvtriflj \\
\hline
\end{tabular}

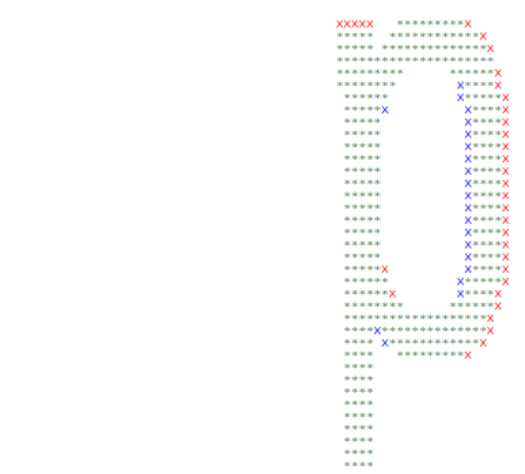

Fig. 3. Green pixels are placed identically in letter $\mathrm{p}$ and rotated letter $\mathrm{q}$ Red and blue pixels differs on matrix. Very high similarity can be seen between these two letters.

2) Scale Invariant Feature Transform (SIFT)

The detection of local features in computer vision is a method of calculating and deciding whether given pixel is important for processing. It is able to detect corners, edges, blobs and ridges.

Its benefit is invariance and robustness. Especially for invariance ability we chose this method for supporting results of our LAP Algorithm. Although SIFT might be for multidimensional vectors time consuming (depending on the size of data), for image comparison is necessary. Comparing the similarity of images is carried out under a certain threshold similarity values. To reduce computational complexity, the descriptors may be filtered, for example to by defining a plurality of descriptors specified area of the display.

Shapes and sizes of local descriptors processed in a program developed at Faculty of Informatics, Masaryk University [26], are shown on Fig. 4 and Fig. 5. Similarity of descriptors is on Fig. 6 (the letters are similar, considering the fact that $q$ can in fact be seen as $p$ rotated $180^{\circ}$ on axis $x$; these letters will be confusing for users with dyslexia) and Fig. 7. The lower ratio difference of compared image to original one is, the higher visual similarity is present.

\section{q z}

Fig. 4. Number of visible descriptors for $q$ is 6 ; number of visible descriptors for $z$ is 18 . The ratio is $1: 3$. The letters are not much visually similar to users with dyslexia.

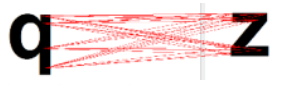

Fig. 5. Showing similar descriptors between $q$ and $z$ on different positions (non-similarity).

\section{p q}

Fig. 6. Number of visible descriptors for $p$ is 6 ; number of visible descriptors for $q$ is 6 . The ratio is $1: 1$.

\section{p $\mathbf{q}$}

Fig. 7. Showing similar descriptors between $p$ and $q$ (similarity).

\section{SUMMARY}

The results of our LAP algorithm partially corresponded with SIFT. Absolute compliance was not expected because SIFT is not designed primarily for determination of letter similarity for dyslexia problem solution; on the other hand, dyslexia is a very individual disorder and, because of this fact, the formalisation and its results cannot be applied absolutely. Significant congruence was found between soft data obtained during the testing of the volunteers and LAP algorithm similarity search; also some results using SIFT algorithm were amenable.

Our research can be beneficial to designers and assistive technology developers. Improving the formalisation (based on our up to date findings) on which we can build an application is a step towards easier web text accessibility.

\section{REFERENCES}

[1] F. Ramus, "Developmental dyslexia: specific phonological deficit or general sensorimotor dysfunction?" Developmental Dyslexia: Specific Phonological Deficit or General Sensorimotor Dysfunction, vol. 13, no. 2, pp. 212-218, 2003 .

[2] J. F. R. Manis, C. Mcbride-Chang, M. S. Seidenberg, P. Keating, L. M. Doi, B. Munson, and A. Petersen, "Are speech perception deficits associated with developmental dyslexia?" Journal of Experimental Child Psychology, vol. 66, no. 2, pp. 211-235, 1997.

[3] K. Plessen, A. Lundervold, N. Duta, E. Heiervang, F. Klauschen, A. Smievoll, L. Ersland, and K. Hugdahl, "Less developed corpus callosum in dyslexic subjects — a structural MRI study," Neuropsychologia, vol. 40, no. 7, pp. 1035-1044, 2002.

[4] T. Athanaselis, S. Bakamidis, I. Dologlou, E. N. Argyrious, and A. Symvonis, "Making assistive reading tools user friendly: A new platform for Greek dyslexic students empowered by automatic speech recognition," Multimedia Tools and Applications, vol. 68, no. 3, pp. 681-699, 2012.

[5] G. Kenvinde, L. Rello, and R. Baeza-Yates, "IDEAL: A dyslexicfriendly ebook reader," in Proc. ACM SIGACCESS Conf. on Computers and Accessibility, 2012, pp. 205-206. 
[6] L. Rello and R. Baeza-Yates, "Evaluation of DysWebxia: a reading app designed for people with dyslexia," in Proc. Web for All Conference, 2012, pp. 1-10.

[7] R. Engel-Eldar and J. Rosenhouse, "Hearing-impaired students' reading characteristics compared to dyslexic students (tested in Hebrew)," International Journal of Rehabilitation Research, vol. 23 no. 4, pp. 313-318, 2000.

[8] L. Rello, C. Bayarri, and A. Gorriz, "What is wrong with this word? dyseggxia: a game for children with dyslexia," in Proc. ACM SIGACCESS Conference on Computers and accessibility, 2012, pp. 219-220.

[9] J. Jošt, Reading and Dyslexia, Grada Publishing, 2011.

[10] B. A. O’Brien, J. S. Mansfield, and G. E. Legge, "The effect of prin size on reading speed in dyslexia," Journal of Research in Reading, vol. 28 , no. 3, pp. 332-349, 2005.

[11] L. Rello and R. Baeza-Yates. (2013). Good fonts for dyslexia. [Online].

Available: http://dyslexiahelp.umich.edu/sites/default/files/good_fonts_for_dysle xia_study.pdf

[12] M. Batko, D. Novák, and P. Zezula, "MESSIF: Metric similarity search implementation framework," Digital Libraries: Research and Development, vol. 4877, pp. 1-10, 2007.

[13] J. Rygl, "Automatic adaptation of author's stylometric features to document types," in Proc. Text, Speech, and Dialogue, 2014, pp. $53-$ 61.

[14] H. Bay, A. Ess, T. Tuytelaars, and L. van Gool, "Speeded-up robust features," Computer Vision and Image Understanding, vol. 110, no. 3, pp. 346-359, 2008.

[15] B. Sirmacek and C. Unsalan, "Urban-area and building detection using SIFT keypoints and graph theory," IEEE Transactions on Geoscience and Remote Sensing, vol. 47, no. 4, pp. 1156-1167, 2009.

[16] L. Chen, M. T. Özsu, and V. Oria, "Robust and fast similarity search for moving object trajectories," in Proc. ACM SIGMOD Int. Conf. on Management of Data, 2005, pp. 491-502.

[17] K. Heikamp, A. M. Wassermann, and J. Bajorath, "Potency-directed similarity searching using support vector machines," Journal of Cheminformatics, vol. 4, no. 1, pp. 12, 2012.

[18] V. Figueredo de Santana, R. de Oliveira, L. Dell Anhol Almeida, and M. C. Calani Baranauskas, "Web accessibility and people with dyslexia: a survey on techniques and guidelines," in Proc. Int. Cross Disciplinary Conf. on Web Accessibility, 2012, Article 35.

[19] S. Pandey and S. Srivastava, "Tiblo: A tangible learning aid for children with dyslexia," in Proc. Conf. on Creativity and Innovation in Design, 2012, pp. 211-220.

[20] A. MacFarlane, A. Albrair, C. R. Marshall, and G. Buchanan, "Phonological working memory impacts on information searching: an investigation of dyslexia," in Proc. Conf. Interaction in Context Symposium, 2012, pp. 27-34.

[21] S. Kim, L. J. Lombardino, W. Cowles, and L. J. Altmann, "Investigating graph comprehension in students with dyslexia: An eye tracking study," Research in Developmental Disabilities, vol. 35, no. 7, pp. 1609-1622, 2014.

[22] J. Doležal and V. Fabian, "41. Application of eye tracking in neuroscience," Clinical Neurophysiology, vol. 126, no. 3, p. e44, 2015.
[23] J. C. Ziegler, C. Perry, A. M. Jacobs, and M. Braun, "Identical words are read differently in different languages," Journal of the Association for Psychological Science, vol. 12, no. 5, pp. 379-384, 2001.

[24] D. G. Lowe, "Object recognition from local scale-invariant features," in Proc. IEEE International Conference on Computer Vision, 1999, pp. $1150-1157$.

[25] D. G. Lowe, "Distinctive image features from scale-invariant keypoints," IEEE International Journal of Computer Vision, vol. 60 , no. 2, pp. 91-110, 2004.

[26] M. Labuda, "A tool for visualization of image descriptors," B. S thesis, Faculty of Informatics, Masaryk University, Brno, Czech Republic, 2012.

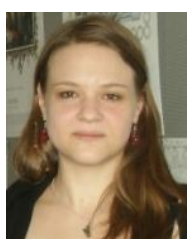

Tereza Pařilová was born in Boskovice, Czech Republic. She received her master degree in 2012 at the Faculty of Informatics, Masaryk University, majoring in service science, management and engineering. For her study results she was awarded the dean prize for outstanding student. She is now pursuing her $\mathrm{PhD}$, focusing on research in assistive technologies at the Faculty of Informatics and in neuroscience at Faculty of Medicine, Masaryk University. She has been working as a lecturer in the Department of Computer Systems and Technologies and cooperates with Central European Institute of Technology. So far she has presented her results in United States, Europe and Japan.

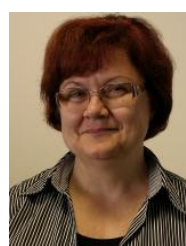

Eva Hladká is an associated professor at the Faculty of Informatics, Masaryk University, as well as a senior researcher at the Czech National Academic Network where she heads the Department Multimedia Transfers and Collaborative Environments. Her research interests include programmable networks, virtual multicast, virtual collaborative environments, multimedia support of teaching and telemedicine. She is in a headboard of the Laboratory of Advanced Network Technologies, joint laboratory of Faculty of Informatics, MU, Institute of Computer science, MU, and CESNET, she has been teaching in the same place. She had been involved in EU projects Ithanet, EuroCareCF and EUAsiaGrid. She is also the author of more than 60 international peer reviewed publications.

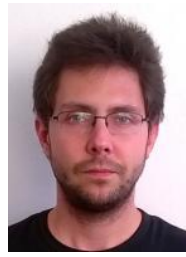

Jaroslav Bayer gained his Dr. Rer. Nat. at the Faculty of Informatics, Masaryk University, majoring in applied informatics and data mining. He has been working within Computer System Units and Figures as a lecture of $\mathrm{C}++$. He has been also an advisor of many bachelor and master diploma theses and works on Masaryk University Information System development. $\mathrm{He}$ cooperates on developing tools for dyslexia users and actively participates in many other projects regarding information and communication technologies. He also publishes regularly wide topics of technological and natural science matter. 